\title{
A Smart Irrigation and Monitoring System
}

\author{
Dishay Kissoon \\ Department of Information, \\ Communication and \\ Technology \\ Faculty of Information, \\ Communication and \\ Digital Technologies \\ University of Mauritius
}

\author{
Hinouccha Deerpaul \\ Department of Information, \\ Communication and \\ Technology \\ Faculty of Information, \\ Communication and \\ Digital Technologies \\ University of Mauritius
}

\author{
Avinash Mungur \\ Department of Information, \\ Communication and \\ Technology \\ Faculty of Information, \\ Communication and \\ Digital Technologies \\ University of Mauritius
}

\begin{abstract}
Internet of Things, commonly known as IoT is a promising area in technology that is growing day by day. It is a concept whereby devices connect with each other or to living things. Internet of Things has shown its great benefits in today's life. Agriculture is one amongst the sectors which contributes a lot to the economy of Mauritius and to get quality products, proper irrigation has to be performed. Hence proper water management is a must because Mauritius is a tropical island that has gone through water crisis since the past few years. With the concept of Internet of Things and the power of the cloud, it is possible to use low cost devices to monitor and be informed about the status of an agricultural area in real time. Thus, this paper provides the design and implementation of a Smart Irrigation and Monitoring System which makes use of Microsoft Azure machine learning to process data received from sensors in the farm and weather forecasting data to better inform the farmers on the appropriate moment to start irrigation. The Smart Irrigation and Monitoring System is made up of sensors which collect data such as air humidity, air temperature, and most importantly soil moisture data. These data are used to monitor the air quality and water content of the soil. The raw data are transmitted to the cloud platform, Microsoft Azure cloud platform, and are processed through a machine learning operation which had to be trained beforehand. The farmer is then informed through either a web app or mobile app as to when to irrigate. The Smart Irrigation and Monitoring System proposed in this paper allows the farmer, through both the mobile app and web app to send command to start the irrigation process.
\end{abstract}

\section{General Terms}

Machine Learning, Internet of Things, Smart Irrigation

\section{Keywords}

Internet of Things, a Smart Irrigation and Monitoring System, Microsoft Azure Cloud, Azure Machine Learning

\section{INTRODUCTION}

Mauritius is a country known for its agriculture and sea [1]. In parallel to growing crops, its irrigation needs must also be taken into consideration. Crops require proper irrigation at appropriate time intervals for them to grow healthily. Agriculture is a field where labour is very crucial and is also in high demand. As revealed in a recent study of the Human Resource Development Council (HRDC), the employment rate in the primary sector has been foreseen to decrease nearly from $8.1 \%$ to $4.4 \%$ by 2015 [2]. The reason behind this decline in work force was because youngsters were not passionate about agricultural sector and they did not find much opportunities. As a result, farmers who dedicate their times to grow crops in large areas had to spend their whole day outside to ensure that the crops are being grown properly. Farmers rarely had good controls on crops and also suffered from great losses due to unforeseen, and inconvenient weather conditions. Mauritius being a country where rain is very unpredictable [3] [17], farmers do not have enough resources to closely monitor their fields so as to know when is the appropriate moment to start irrigating their crops. There are situations where farmers irrigate their crops without knowledge of the weather forecast. As a result, this situation leads to bad water management. Thus, this paper proposes a Smart Irrigation and Monitoring System (SIMS) which uses sensors to collect air humidity, air temperature, and most importantly soil moisture, and transmit the raw data to a cloud platform (Microsoft Azure cloud platform). The data are then processed, using Azure Machine Learning Algorithms (the two-class boosted decision tree algorithm has been selected to train and process the data). The algorithm also factors in weather forecast data in order to provide an informed decision to the farmer when irrigation is required. The farmer is notified about the decision whether to irrigate or not through a either a web app or mobile app which is developed using Xamarin. Based on the decision received from the machine learning process, the farmer can trigger the irrigation process through his mobile phone. Same is also provided through a web interface.

This paper is organized as follows: Section 2 provides a literature review in the domain of smart farming particularly geared towards irrigation along with a critical analysis. Section 3 provides an overview of the proposed architecture. Section 4 provides the evaluation of the proposed architecture. Section 5 concludes the paper and section 6 is the acknowledgement section.

\section{LITTERATURE REVIEW}

This section provides a general survey of the domain (as carried out in [4], [5], [6], [7], and [8]) and a critical analysis of the work is provided.

In [4], the authors describe IoT as being the gateway of communication among things. It also highlights the importance of agriculture in Thai economy. The main target of the authors was to develop a watering and roofing system for outdoor agricultural sites. The system comprised mainly of data where physical factors such as moisture are provided by sensors. The system focuses more on the concept of Kalman filtering to remove noise from one sensor to another for obtaining more accurate values. The authors make use of a decision tree model to determine when it is appropriate to start watering using certain standards as shown in Table 1. A mobile application has been developed where the user can see 
the sensor data from the cultivation field, and can control the watering and roofing system directly from his phone.

Critics: This paper assesses mainly the physical data from sensors and then compare them with weather forecast by the use of a decision tree model whose results are shown in Table 1 .

Table 1. Results from Decision Tree Model

\begin{tabular}{|c|c|c|}
\hline $\begin{array}{c}\text { Result from } \\
\text { Decision tree }\end{array}$ & $\begin{array}{c}\text { Smooth sensed } \\
\text { data }\end{array}$ & Decision to do \\
\hline "no rain" & Mositure $\geq 70 \%$ & "watering" \\
\hline "no rain" & $\begin{array}{c}\text { Moisture } \leq 70 \%, \\
\text { Light } \leq 2,000 \text { lux } \| \\
\text { Temp } \leq 35 \text { Celsius }\end{array}$ & "closing roof" \\
\hline "rain" or "storm" & Mositure $\geq 70 \%$ & $\begin{array}{c}\text { "wait for } \\
\text { raining" }\end{array}$ \\
\hline "rain" or "storm" & Mositure $\geq 70 \%$ & "opening roof" \\
\hline
\end{tabular}

From Table 1, it can be observed that an optimum value of $70 \%$ has been used for moisture, 2000 lux for light and 35 degrees Celsius for temperature. As stated in the paper, these values are that of cabbage. Therefore, different plants have different optimum conditions. The decision tree model has been devised to allow the author to take decision for watering the crops. The author uses node.js library in order to compute the decision. The node.js library used by the authors is an integration of machine learning libraries. However, the node.js library cannot be considered as a full-fledged processing system like Azure Machine learning since it does not provide an array of algorithms which can be used to generate the prediction. Microsoft Azure machine learning provides different algorithms that are readily available and the appropriate one can be chosen and configured or adapted to suit the requirement. Furthermore, the author has not mentioned the type of soil on which they are experimenting. The type of soil is important since different type of soils requires different types of irrigation process. A notification alert could also have been implemented so as to notify the user about any particular changes in the crop area. Since the author is dealing with graphs, a web application could have been developed to have a better view of the sensor values.

In [5], the paper proposes a smart irrigation system with the use of IoT together with smart technologies. Taking into consideration the wrong usage of water in the southern Algeria, a smart irrigation system that can be controlled and monitored has been devised to manage the usage of water more efficiently. The irrigation system is ensured to be costeffective and detailed. Wireless Sensor Network (WSN) and IoT technologies have been used to develop the system. Constrained Application protocol (CoAP) and web application have been used to complete the implementation of the system where the web application was mainly for controlling the irrigation through the Internet.

Critics: The project was mostly based on the network connections, that is, it has focused more on establishing the WSN. Though it is good to explore different kinds of ICT technologies, the system could have been simpler. This is because the system can target the majority of the population with its simplicity. A database system could also have been established if historical sensor data need to be reviewed later on. In addition, the system could have used microcontrollers or microprocessors to make the connection between the irrigation area and the mobile application easier.

In [6], the work is based upon an efficient and friendly Arduino-based automatic irrigation system which makes use of Android smart phone for remote control. The prototype consists of a soil moisture sensor that gives a voltage signal proportional to the moisture content in the soil which is correlated with a fixed threshold value retrieved by inspecting various soils and explicit crops. As a result, the convenient data are sent to the Arduino Uno processor which is connected wirelessly through the HC-05 module to an Android smart phone. The data obtained is displayed on the User Interface of the Android smart phone. In this case, the remote control in the Android smart phone is used to manage the irrigation drive system by switching it on and off. The system has proven to be feasible and can be readily used for real time application.

Critics: The system was based upon an automated irrigation system by using mainly a soil moisture sensor and an Android smart phone. With this system, people can have a better control on their irrigation time and can also save water. In this prototype, different soil samples and crops for calibration at various moisture levels was tested. However, to improve this analysis, various soil samples from different places could have been tested and also during different weather conditions. Apart from soil moisture, other factors of the soil could have also been monitored.

In [7], IoT has been used in order to analyze sensor devices which take in some physical information and address it back to the user. The paper is based on highlighting the methods used to solve problems such as recognition of rodents, risks to crops, and transfer of real time notification without the interference of human beings. Python scripts were used to incorporate the sensors and electronic devices.

Critics: In this paper, IoT was used to improve the security of crops from pests and insects in grain stores. IoT together with the security devices were beneficial in the sense that they allowed information transmission and data analysis in order to get adequate food preservation and productivity. Machine to machine (M2M) framework, sensor network, and database management are the root factors behind this IoT based intelligent security device. M2M is beneficial in the way that it has a strong industrial significance. M2M is a platform that works considerably well with IoT and there are also lots of resources available for the combination of M2M/IoT. Extended "as-a-Service" framework has also been suggested to work together with IoT in order to provide a more costefficient project. PIR sensors (used mainly to sense motion) have been used in this project to detect heat. However, this sensor does not get along well with raspberry pi. Arduino could have been used instead of the raspberry pi to cater for the compatibility with the sensor and in addition Arduino is cheaper as compared to raspberry pi.

In [8], the concept of IoT and web services have been used in order to professionally handle the huge data involved in the cultivation of land. Using the combination of IoT and cloud computing, modern agriculture has evolved quickly and has also contributed to develop smart solutions for agriculture. It also caters for the problems face by farmers productively.

Critics: The paper gives a brief idea of an experimental model of how IoT can be used to enhance agriculture field. A model blueprint of how IoT concepts have been suggested to be used in agriculture for better productivity. DHT11 sensors have 
been used in this system to predict both temperature and humidity simultaneously. ZigBee is a system that caters for the standardization of IoT by providing an authoritative standard to the equipment used in WSNs. The ZigBee sensor nodes which have been used in the prototype can detect natural factors such as temperature, humidity and light which are then addressed to the remote monitoring center. The sensor nodes and the data centers are connected by the CDMA, 3G, and 2G wireless broadband networks. WLAN 802.11 and Bluetooth are used for communications of nearest node. The sensor control module is liable for tracking the incoming information and process appropriately. Apart from all these, a cloud system could have been implemented in this prototype in order to have a better analytics and control on the system.

After having reviewed the different research work, this paper proposes a Smart Irrigation and Monitoring System which provides farmers with real time insights of their crops through the use of a cloud platform and machine learning (taking into account weather forecast for that region). A low-cost system has been proposed so as to make it available to everyone. Following that, 24/7 farm monitoring is provided through a collection of sensors in order to have a better control of the soil moisture content, air humidity, and air temperature. The data is then transformed into a more meaningful way like graphs. The prediction is then generated by comparing the raw sensor data with weather forecast through a machine learning process. The weather forecast is obtained from Openweathermap.com since it provides free weather API [9]. However other weather API can also be integrated and not limited only to Openweathermap.com.

The results obtained from the machine learning processing will enable farmer to take an informed decision as to when the water tap needs to be opened, that is, the farmer is informed about the probability of rain and whether he should irrigate or not. Consequently, the farmer is able to send a command to open the water taps remotely through a mobile application or web app. This will enable the farmer to save valuable time in terms of starting the irrigation process and thus water will be better managed. A web app has also been developed in order to have a better view about the statistical data of the sensor values by means of graphs and also for any inquiry purposes about the application. A dashboard has also been devised to control the system through the web app.

\section{PROPOSED SYSTEM ARCHITECTURE}

In this section, the proposed system architecture is elaborated. Figure 1 shows an architectural overview of the proposed system. The proposed architecture for the Smart Irrigation and Monitoring System has been divided into 6 main components namely: Physical Environment (A), Azure IoT Hub (B), Stream Analytics (C), Azure SQL Database and Power BI (D), Azure Machine Learning (E), and the mobile application (F).

\subsection{Physical Environment}

The first part is the plant section where sensors (DHT22, YL100 Soil Moisture) are connected on a WeMos Board [10] for sensing the plant's surroundings. The DHT22 is a costeffective sensor that is used for monitoring both the air temperature and the humidity of air [11]. YL-100 soil moisture sensor [12] is used to measure the soil moisture content. The moisture sensor outputs a high level when the soil lacks water otherwise it outputs a low level. Besides, the sensitivity of the soil moisture sensor is flexible and unlike other soil moisture sensors, the YL-100 sensor does not require any additional convertor to operate. As illustrated in figure 1, the sensors feed in raw data from the plant's environment to the WeMos Board which then sends the raw data from sensors to Azure IoT Hub.

\subsection{Azure IoT Hub}

The Azure IoT Hub is a service that allows bi-directional communication among devices. The Azure IoT hub acts as a middleman between different services and the physical environment, that is, device to cloud communication and vice versa. It receives updated data from sensors regularly. In general, the job of the Azure IoT Hub is mainly for monitoring every IoT devices and link them together.

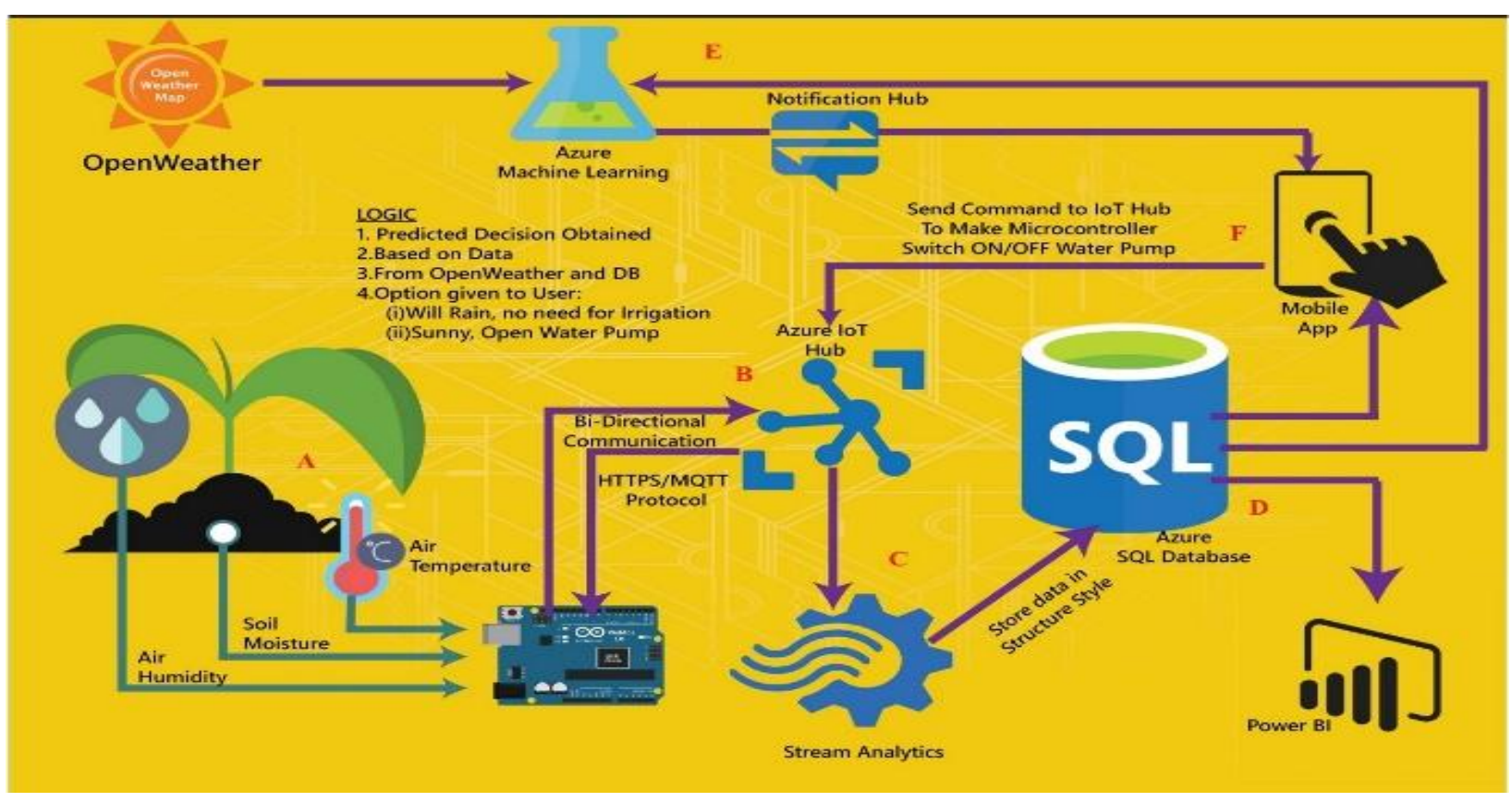

Fig. 1. Proposed Architecture 


\subsection{Stream Analytics}

Stream Analytics is a service offered by Azure and is a mandatory path to pass on data from the Azure IoT Hub to the Azure SQL Database. Intrinsically, the core feature of this service is to provide the flexibility of streaming millions of records per second. The raw data is sent in the form of JSON format and is structured into a tabular form so that it can be stored into the Azure SQL Database.

\subsection{Azure SQL Database and Power BI}

At this point, the data found in the database needs to be transformed into a more user-friendly representation as farmers will not be understand SQL queries. Hence, to cater for this problem, Power BI is used to reconstruct the data into a visual representation such as a graph

\subsection{Azure Machine Learning}

The Azure machine learning is the core logic of the proposed system. In general, a dataset is needed to train the machine to find patterns in the data in order to decide whether to irrigate or not. For better precision, a Openweathermap.com API is included with the aim of knowing when the water pump needs to be opened. The pseudocode in figure 2 gives a simple illustration on how the machine learning system works.

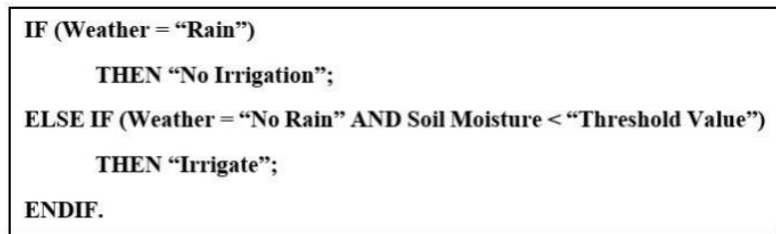

Fig 2: Pseudocode

In machine learning, everything is about modules, that is, machine learning works through a chain of modules. For machine learning to work, it should be trained through data and patterns. In the proposed machine learning system, a "training experiment" has been used and figure 3 shows this concept.

\subsubsection{SmartFarmingData Module}

The dataset is input in this module. Basically, it contains a variety of data and need to be clarified to obtain the desired data, that is, the data for soil moisture, air humidity, and air temperature. The clarification is performed by removing null dataset. The dataset chosen is based on a chart.

\subsubsection{Select Column in Dataset Module}

This module is implemented in the chain so as to be able to train the machine. Specific column has to be selected in order to do the prediction on a specific parameter.

\subsubsection{Split Data Module}

The split data module is divided into two parts: mainly the Train Model and The Score Model (See section 3.5.4).

The Train Model module contains $70 \%$ of the dataset for the machine learning. The required pattern is trained in this area and to do so, an algorithm needs to be derived for it to know which data the pattern should consist of. The algorithm used is known as a 'Two-class boosted decision tree' and lies under the 'two-class classification' algorithm structure. Figure 4 shows the different algorithms for the "two-class classification' algorithms. It can be observed that there are nine types of algorithms in this category and they differ from each other. As illustrated in figure 4, the 'Two-class SVM' has hundred features and provides a linear model, the 'Twoclass decision forest' provides accuracy and fast training and the 'Two-class neutral network' provides accuracy in addition to long training times. The 'Two-class boosted decision tree' has been selected in this paper because the algorithm is known for its accuracy, fast training and large memory footprint [13]. With these facilities, a lot of data can be passed quickly without any difficulty and large dataset can be stored. Furthermore, since one decision needs to be taken, that is, either to irrigate or not, this algorithm suits the proposed system.

SmartFarmingML

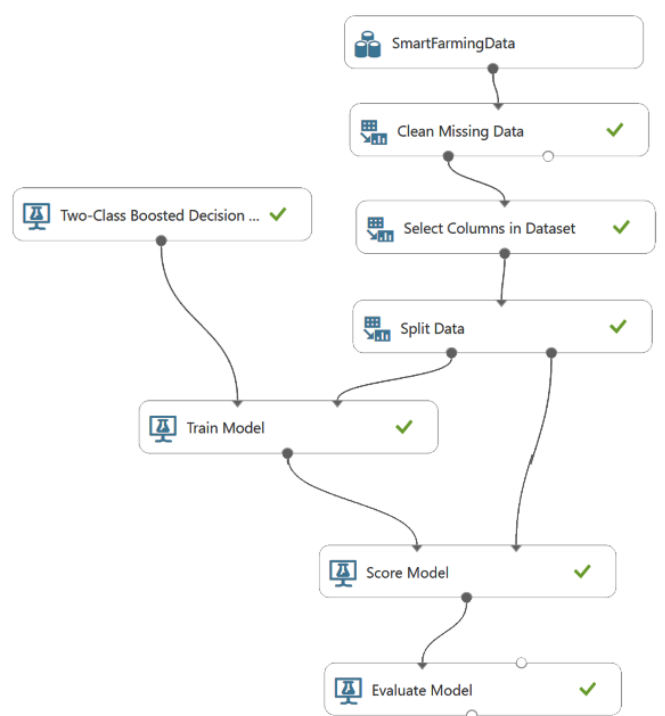

Fig 3: Training Experiment of Machine Learning

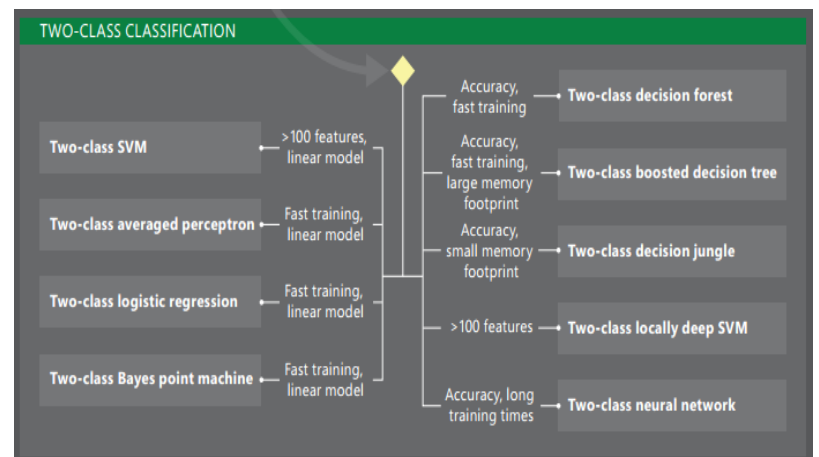

Fig 4: Two-class Classification (Microsoft, 2017) [13]

\subsubsection{How the machine learning process the prediction?}

The Azure machine learning process occurs in two steps; first being the comparison between the raw data with that of a threshold value and the second is comparing the result from the first step with that of weather forecast. Normally, a threshold value is a value which on exceeded will cause a change in the action of a particular thing. In this instance, the threshold value should base on the soil moisture. That is the moment at which the soil requires watering. In general, the threshold value could be set based on the graph as suggested in [14]. The latter provides a range of soil moisture value which can used for the threshold value, upon which irrigation can start. However, the value suggested in [14] is not appropriate for Mauritian soil.

According to [15], there are six main types of soil namely: Sandy, Peaty, Loamy, Clay, Silty and Chalky. The type of soil 
present in Mauritius is considered to be thick clay or basalt soil [17] [18]. Clay soil is known to be lumpy and sticky when very wet and hard like rock when dry. It also drains poorly and contains little air space. It is also heavy to cultivate into clay soil and if drainage is properly managed, the plants are considered to grow well. Hence according to [14], the threshold value for irrigation for clay soil is $33.0 \%$. However, after having collected soil moisture value in the region of Coromandel, Mauritius, the threshold value for irrigation for this region is $27.0 \%$. Hence, the threshold value needs to be adapted based on the region's soil moisture.

After comparing the raw data with the threshold value (27.0\%), the Azure machine learning will then compare the result with historical weather forecast from
Openweathermap.com and then predict whether irrigation needs to be done.

\subsubsection{Score Model Module}

After training the dataset, the machine learning checks whether the predicted value is accurate enough. To do so, a score model module needs to be set and it is based on the $70 \%$ dataset that has been provided from the Train Model. A random value is passed into the machine learning and is linked to the remaining $30 \%$ of data to perform the prediction.

\subsubsection{Evaluate Model Module}

This module has been included during the testing mode and is intended to provide a more user-friendly representation of the results predicted.

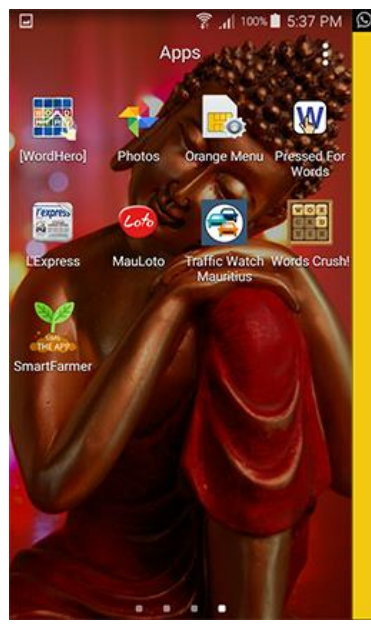

\subsection{Mobile Application}

After processing all the raw data on the cloud platform, the user would be able to view them and the predictions through a mobile application. The interface of the mobile application is shown in figure 5 . The mobile application contains a navigation drawer that has as default a weather forecast page and provides the farmer with a list of choices. The Arduino Registration allows the user to register his device for recognition purposes on the Azure IoT Hub and the farmer is provided with a unique Key. This registration is important in cases where multiple Arduino boards will be used and hence each of these microcontrollers need to have their own identification. The Sensor Readings and Graphs are used to view statistical data that has been processed. Lastly in the Action menu the prediction from the machine learning is displayed and options to open the water pump with the switch ON/OFF button is provided.

Xamarin Studio has been used to develop the mobile application as it is cross platform and hence it can target a maximum number of users. Broadly, the mobile application notifies the user to open the water pump if irrigation needs to be done. The user will have the flexibility not to irrigate if he feels that it is not necessary to irrigate even if he has been notified to open the water pump. Assuming that the user chooses to irrigate, once the button "ON" is clicked on the mobile application, the signal will be sent to Azure IoT Hub which will in turn forward the signal to the WeMos board to open the water pump for irrigation.

\subsection{The Web App}

The web app is where the functions from the Power BI will figure in. The web app has been designed using open source web design template. The reason for choosing open source is simply because it is free and we are exposed to the source code and hence we only need to amend the template as per our requirements. The web app has been developed in the waterfall development process. Figure 6 shows a picture collage of the web app. The web app will consist of the Power BI where the graphs of the sensor data appears. The graphs will be fully discussed in section 4 . The 'sensors' part in the web app contains information about the sensor. By hovering on the pictures, the latest sensor value will be displayed.

\section{EVALUATION}

Figure 7 shows the sensor readings that have been streamed into JSON format to the Azure IoT Hub. Device Explorer [16] has been used to view the records that are being streamed every thirty seconds. The system was then programmed to stream data (soil moisture, air humidity and air temperature) regularly. From figure 7, it can be seen that the following data has been streamed: Date, Device ID (Mega2560), Data (Record ID, Time, Temperature in Celsius, Air Humidity, Soil Moisture, Geo-Location of the board). The sensor readings obtained from figure 7 are then transformed into graphs with the use of Power BI through the web application. Figure 8 shows the temperature sequence, the soil moisture values and the humidity readings that have been generated through the Power BI. 

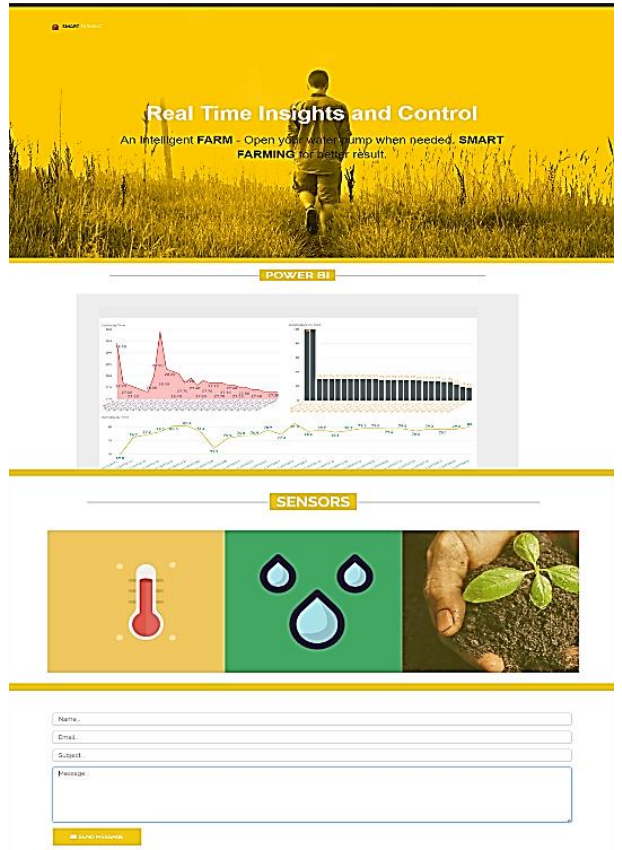

Fig. 6. Screen Interface of the Web App

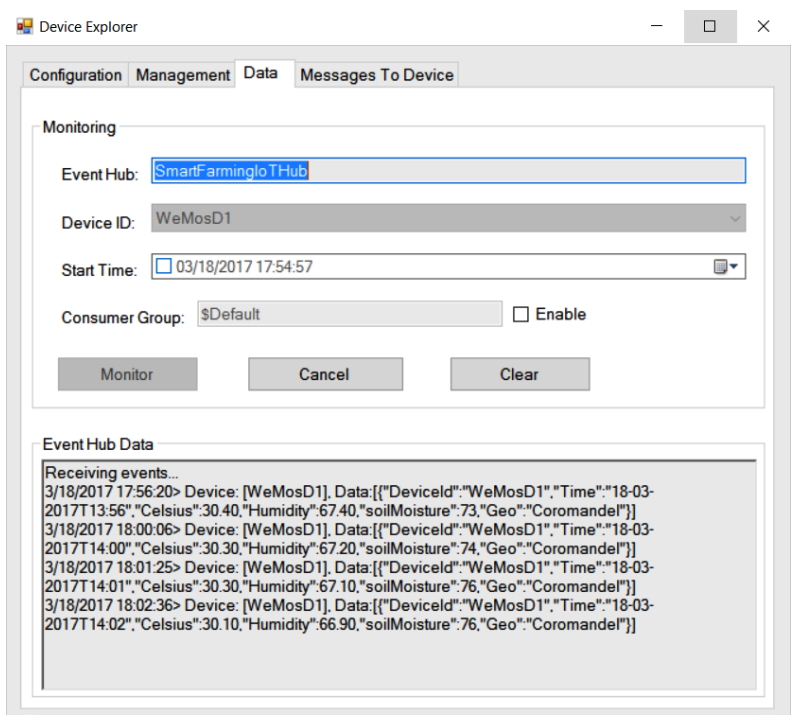

Fig. 7. Sensor Reading

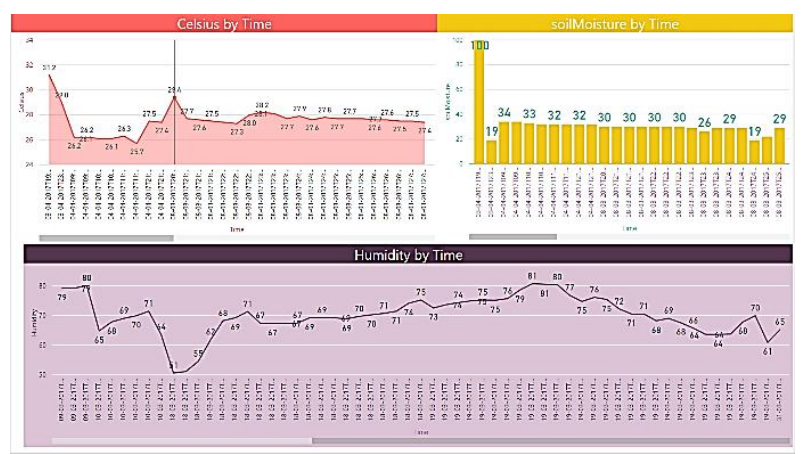

Fig. 8. Graphs Representing the Sensor Values

The graphs produced in figure 8 respond dynamically to the users, that is, when a particular point is selected from any of these three graphs, the value will be automatically displayed. Figure 9 shows the dynamic response of the graphs.

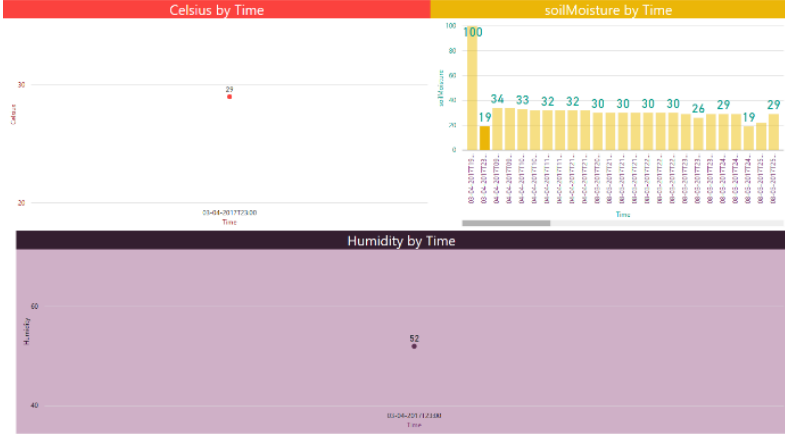

Fig. 9. Dynamic Response of Graphs

Figure 10 shows the results from the Azure machine learning. In figure 10, the sensor data values can be observed and the predicted irrigation choice has been shown. The scored labels show the predicted values and the scored probabilities displays the accuracy of the predicted value. Based on the value of the "Irrigate" column, the user will be notified about the outcome. The user will then decide whether to irrigate or not.

\begin{tabular}{|c|c|c|c|c|c|c|}
\hline \multirow[t]{2}{*}{$\begin{array}{l}\text { rows } \\
60\end{array}$} & \multicolumn{6}{|l|}{$\begin{array}{l}\text { columns } \\
6\end{array}$} \\
\hline & Air Temprature & Air Humidity & Soil Moisture & Irrigate & Scored Labels & Scored Probabilities \\
\hline \multirow[t]{14}{*}{ 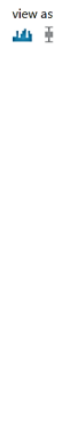 } & |.|l||l.|| & $\|$ |ndlun & |.|.||l||l|| & $\square_{a}$ & I. & \\
\hline & 29.63 & 67.58 & 20 & yes & yes & 0.978723 \\
\hline & 29.7 & 67.32 & 29 & no & no & 0.010417 \\
\hline & 27.89 & 74.76 & 26 & no & no & 0.010417 \\
\hline & 31.29 & 61.46 & 38 & no & no & 0.010417 \\
\hline & 26.87 & 79.41 & 31 & no & no & 0.010417 \\
\hline & 28.57 & 71.88 & 28 & no & no & 0.010417 \\
\hline & 27.08 & 78.39 & 36 & no & no & 0.010417 \\
\hline & 27.84 & 75.01 & 27 & no & no & 0.010417 \\
\hline & 27.58 & 76.14 & 25 & no & no & 0.010417 \\
\hline & 30.96 & 62.66 & 39 & no & no & 0.010417 \\
\hline & 28.45 & 72.37 & 16 & yes & yes & 0.978723 \\
\hline & 27.91 & 74.69 & 30 & no & no & 0.010417 \\
\hline & 31.08 & 62.22 & 37 & no & no & 0.010417 \\
\hline
\end{tabular}

Fig. 10. Results from Azure Machine Learning

\section{CONCLUSION}

A Smart Irrigation and Monitoring System has been proposed so as to reduce wastage of water and to automate the irrigation structure of large areas of crops. The system mainly monitors the behavior of soil moisture, air humidity, and air temperature and see how it contributes to evaluate the needs of water in a plant. The system uses machine learning and compares actual values obtained from sensors with a threshold value that has been fed to the machine learning for analysis. After this process, the machine learning cross checks the result obtained with weather forecast and then decides whether irrigation needs to be done or not. The farmer receives a notification on his smart phone and he can choose to turn on the water pump with a button click. Moreover, the system has a web app and is helpful if ever the farmer wants to see the statistical sensor data and assess the change in sensor readings throughout a time period. Furthermore, the system can be calibrated for different type of plants, that is, the user is provided with a list of plant's choices in his web app and mobile app. With this the user can choose the specific type of plant that is being cultivated and obtain a more precise threshold value and thus a more accurate irrigation prediction. Besides, an SMS system can be integrated if in case there is no internet connection. With this, the user would be notify about the prediction via an SMS and he can choose to switch on or off the water pump by replying to the SMS received. 


\section{ACKNOWLEDGEMENT}

We would also like to show our greatest appreciation to Dr. L. $\mathrm{Ng}$. Ronal Cheong from the Mauritius Sugar Industry Research Institute (MSIRI) for guiding us about the soil factors and different plant types in order to set our prototype for the system.

\section{REFERENCES}

[1] Travel Guide, Mauritius Travel Guide, [Online] http://www.mauritiusholidaystips.com/wpcontent/uploads/2011/04/Mauritius-Activity-guide.pdf

[2] Human Resource Development Council, 2012. A Study on Labour Shortage in the Agricultural Sector in Mauritius, October 2012.

[3] Mauritius Meteorological Services 2017, Climate of Mauritius, [Online] http://metservice.intnet.mu/climateservices/climate-of-mauritius.php

[4] P. Narayut, P. Sasimanee, C.-I. Anupong, P. Phond and A. Khajonpong, 2016. A Control System in an Intelligent Farming by using Arduino Technology. Student Project Conference (ICT-ISPC), 2016 Fifth ICT International, pp. 53-56, 2016.

[5] K. Benahmed, A. Douli, A. Bouzekri, M. Chabane and T. Benahmed, 2015. Smart Irrigation Using Internet of Things. Fourth International Conference on Future Generation Communication Technology (FGCT), 2015.

[6] A. A. N. and K. D, 2016. Experimental investigation of remote control via Android smart phone of arduinobased automated irrigation system using moisture sensor. 3rd International Conference on Electrical Energy Systems (ICEES), 2016.

[7] T. Baranwal, N. and P. K. Pateriya, 2016. Development of IoT based Smart Security and Monitoring Devices for Agriculture. 6th International Conference - Cloud System and Big Data Engineering (Confluence), 2016.

[8] G. M.K., J. J. and A. M. G.S, 2015. Providing Smart Agriculture Solutions to Farmers for better yielding using IoT. IEEE Technological Innovation in ICT for Agriculture and Rural Development (TIAR), 2015.
[9] Openweathermap.com 2012-2016, [Online] http://openweathermap.org/api

[10] Elementzonline 2016. Programming ESP8266 WeMos D1 R2 using Arduino IDE, Random Codes - Elementz Tech, 2016

[11] Adafruit, DHT22 temperature-humidity sensor + extras, [Online] https://www.adafruit.com/product/385

[12] Super Electronics, 2014. Moisture Sensor YL-100 Sensor Kelembapan Tanah Murah, [Online] http://tokosuperelectronics.com/moisture-sensor-yl-100sensor-kelembapan-tanah-murah/

[13] Microsoft, 2017. Machine learning algorithm cheat sheet, Microsoft Azure, [Online] https://docs.microsoft.com/enus/azure/machine-learning/machine-learning-algorithmcheat-sheet

[14] Dynamax, 2017. Soil Moisture Range Chart, Turf Irrigation [Online] ftp://ftp.dynamax.com/turf_irrigation/Soil\%20Moisture\% 20Range\%20Chart.pdf

[15] A. Salam and M. C. Vuran, 2016. Impacts of Soil Type and Moisture on the Capacity of Multi-Carrier Modulation in Internet of Underground Things. 25th International Conference on Computer Communication and Networks (ICCCN), 2016.

[16] GitHub 2017. Azure/azure-iot-sdk-csharp, [Online] https://github.com/Azure/azure-iot-sdkcsharp/tree/master/tools/DeviceExplorer

[17] V. Proag, 2006. Water Resources Management in Mauritius, European Water, E.W. Publications, 2006.

[18] L. Ronald Ng Cheong and Gunshiam Umrit, 2015. Changes in Soil Properties with Sugarcane Cropping in Mauritius, in Land-Use Change Impacts on Soil Processes: Tropical and Savannah Ecosystems, Editor: Francis Q. Brearley, 2015 